\title{
Bmil Severs as a Potential Tumor-Initiating Cell Marker and Therapeutic Target in Esophageal Squamous Cell Carcinoma
}

\author{
Xiaochen Wang, ${ }^{1}$ Kang Li, ${ }^{1}$ Maosheng Cheng, ${ }^{1}$ Ganping Wang, ${ }^{1}$ Hui Han, ${ }^{1}$ Fangfang Chen, \\ Wenjing Liao, ${ }^{1}$ Zhi Chen, ${ }^{1}$ Jianwen Chen, ${ }^{1}$ Yong Bao $\mathbb{D},{ }^{2}$ Liang Peng $\mathbb{D},{ }^{3}$ \\ and Demeng Chen $\mathbb{D}^{1}$ \\ ${ }^{1}$ Center for Translational Medicine, Institute of Precision Medicine, The First Affiliated Hospital, Sun Yat-sen University, \\ Guangzhou 510030, China \\ ${ }^{2}$ Department of Radiation Oncology, The First Affiliated Hospital of Sun Yat-sen University, Guangzhou 510030, China \\ ${ }^{3}$ Oncology Department, Chinese PLA General Hospital, Beijing 100000, China
}

Correspondence should be addressed to Yong Bao; baoyong@mail.sysu.edu.cn, Liang Peng; pengliang_301@163.com, and Demeng Chen; chendm29@mail.sysu.edu.cn

Received 21 April 2020; Revised 6 June 2020; Accepted 10 June 2020; Published 20 August 2020

Academic Editor: Quan Yuan

Copyright ( 2020 Xiaochen Wang et al. This is an open access article distributed under the Creative Commons Attribution License, which permits unrestricted use, distribution, and reproduction in any medium, provided the original work is properly cited.

Esophageal squamous cell carcinoma (ESCC) is a frequent malignant tumor with low 5-year overall survival. Targeting ESCC tumor-initiating cells (TICs) may provide a new research avenue to achieve better therapeutic effects of ESCC. However, the identity and characteristics of ESCC TICs remain poorly understood. Through genetic lineage tracing approach, we found that a group of Moloney murine leukemia virus insertion site 1- (Bmil-) expressing cell populations present in the invasive front of the esophageal epithelium, providing a continuous flow of tumor cells for ESCC. Subsequently, we found that ablation of Bmil ${ }^{+}$ cells from mice with ESCC led to inhibition of tumor growth. In addition, our results demonstrated that PTC-209, an inhibitor of Bmil, was able to inhibit ESCC progression when combined with cisplatin. In summary, our data suggest that Bmi1 ${ }^{+}$cells serve as TICs in ESCC.

\section{Introduction}

Esophageal cancer is one of the most commonly diagnosed cancers, ranking the sixth cancer-related mortality worldwide [1]. It is mainly composed of two histological types: esophageal squamous cell carcinoma (ESCC) and esophageal adenocarcinoma (EAC). ESCC accounts for more than 90\% of the whole esophageal cancer cases in China [2]. However, early diagnosis of ESCC is hard to achieve, resulting in a majority of the ESCC patients diagnosed at advanced stages. And the five-year survival rate of ESCC patients remained around $10 \%$ owing to high recurrence and distant metastasis [3]. Recently, studies have shown that tumor-initiating cells (TICs) or cancer stem cells are the main cause of tumor recurrence and metastasis $[4,5]$. Hence, understanding TICs in ESCC might provide some novel insights in how to improve current treatment of ESCC.

TICs are the cells that are able to self-renew and evolve into the heterogeneous lineages of cancer cells that make up the tumor population [6]. TICs were first identified in leukemia, which have shown that only a limited proportion of transplanted primary tumor cells could cause secondary tumors [7]. Since then, TICs have been successfully isolated from multiple solid tumors by fluorescence-activated cell sorting (FACS) and cell surface markers [8-10]. In ESCC, CD44 is the major marker used in isolation and detection of TICs. For example, CD $44^{+} / \mathrm{CD} 24^{-}$ESCC cells exhibited higher oncogenous potential in vivo [11]. In addition, selecting cells using both $\mathrm{CD} 44$ and ALDH can increase the enrichment effect of TICs by more than 10 times [12]. Other 
surface proteins, such as integrin $\alpha 7$, CD90, and Cripto-1, have been reported as potential TIC markers in ESCC [13-15]. However, traditional transplantation assays often disrupt the native microenvironment of TICs and led to alteration of TIC characteristics [16]. To avoid the shortcomings of these assays, lineage tracing of genetically labeled cells has been used to identify TICs in vivo [17-19].

Moloney murine leukemia virus insertion site 1 (Bmil) is the core component of the polycomb repressive complex 1 (PRC1), which mediates gene silencing through monoubiquitination of histone $\mathrm{H} 2 \mathrm{~A}[20,21]$. Bmil functions as a significant stem cell self-renewal factor. It is also involved in multiple tumorigenic processes, including cell migration, invasion, epithelial-mesenchymal transition (EMT), and chemotherapy resistance $[22,23]$. The expression of $\mathrm{Bmil}$ is associated with the progression and invasion of ESCC [24]. Moreover, it is a potential biomarker for the early diagnosis of ESCC [25]. Importantly, the expression of Bmil in p75 ${ }^{\mathrm{NTR}}$-positive ESCC TICs was higher than that in p $75^{\mathrm{NTR}}$-negative cells [26], which indicates the stemness feature of Bmil. Study has shown that downregulation of CD44 and Bmil in ESCC TICs by administration of nontoxic AUR improved the effect of chemotherapy [27]. Based on these researches, we speculate that Bmil ${ }^{+}$tumor cells might mark CSCs and provide a novel therapeutic molecular target in ESCC.

This article shows that $\mathrm{Bmil}^{+}$cells can represent TICs in ESCC and analyzes the related therapeutic value. For this purpose, we used a mature genetic lineage tracing technique, in which the mice with ESCC were induced by 4nitroquinoline (4NQO). We found that (1) the gene ablation of Bmil led to increased apoptosis, decreased proliferation, and weakened stemness of ESCC; (2) the Bmil ${ }^{+}$tumor cells led to the progressive growth of epithelial clones and the $\mathrm{Bmil}^{+}$tumor cells were tumor-initiating cells in ESCC; and (3) the cisplatin combined with Bmil targeting drug could effectively inhibit tumor growth in ESCC.

\section{Materials and Methods}

2.1. Animal Assays. All animal use protocols and experiments have been approved by the Institutional Animal Care and Use Committee (IACUC), Sun Yat-sen University. The approval number is SYSU-IACUC-2019-000077. All the animal experiments were carried out in the Laboratory Animal Center, Sun Yat-sen University.

BmilCreER, RosatdTomato and Rosa $a^{\text {tTA }}$ mice were obtained from The Jackson Laboratory. To induce ESCC formation in mice, 4NQO (Sigma, N8141) was prepared with 1,2-propanediol (Sigma, 8223245000) into a $10 \mathrm{mg} / \mathrm{mL}$ stock solution and diluted with water for 20 times. The mice aged 8 weeks were fed for 16 weeks and drank normal water for 4 weeks. For lineage tracing, after 16 weeks of 4 NQO treatment, in BmilCreER;Rosa ${ }^{\text {tdTomato }}$ or BmilCreER;Rosa ${ }^{\text {tdTomato }}$;Ro$s a^{D T A}$ transgenic mice, tamoxifen $(0.08 \mathrm{mg} / \mathrm{g}$ body weight per day for 3 days; Sigma, T5648-1G) was injected intraperitoneally. Esophageal samples were collected at different time points and frozen sectioned. The expression and distribution of tdTomato fluorescence protein were analyzed under a fluorescence microscope. For drug treatment assay,
BmilCreER;Rosa ${ }^{\text {tdTomato }}$ mice with ESCC were divided into four different treatment groups, including control group, cisplatin ( $1 \mathrm{mg} / \mathrm{g}$ mouse weight every week; Sigma, BP809) treatment group, PTC-209 (1 mg/g mouse weight every week; MCE, HY-15888-5 mg) treatment group, and cisplatin $(0.5 \mathrm{mg} / \mathrm{g}$ mouse weight every week $)+$ PTC-209 $(0.5 \mathrm{mg} / \mathrm{g}$ mouse weight every week) treatment group. After 4 weeks of drug treatment, ESCC samples were collected and analyzed.

2.2. Hematoxylin and Eosin Staining. The specimens of ESCC with the adjacent mucosal tissues were fixed in $4 \%$ paraformaldehyde (biosharp, BL539A) for up to $24 \mathrm{~h}$ and embedded in paraffin. Then, the section with a thickness of $5 \mu \mathrm{m}$ was cut from the paraffin block and stained with hematoxylin eosin (H\&E) kit following the manufacturer's instruction (Solarbio, G1120-100). Briefly, after heating at $65^{\circ} \mathrm{C}$, the paraffin sections were dewaxed in xylene and hydrated by serial washing in graded ethanol and distilled water, followed by staining in hematoxylin for 5 minutes, color separation with $1 \%$ hydrochloric acid alcohol for 5 seconds, and staining in eosin for 2 minutes. Then, the paraffin sections were dehydrated with graded series of ethanol, removed with xylene, and finally sealed with neutral balsam (Macklin, 822941-100 g).

2.3. Immunohistochemistry (IHC) Staining. Dewaxed paraffin sections were obtained by the same method as mentioned above. Sections were treated with heat-induced epitope recovery with sodium citrate buffer (Bioss, C02-02002), followed with blocking endogenous peroxidase prior to primary antibody incubation. Specific primary antibodies including CD44 (1:200; Abcam, ab157107), Tp63 (1:200; Abcam, ab53039), and KRT5 (1:200; Abcam, ab52635) were used. Horseradish peroxidase (HRP) conjugate was used for DAB staining. Expression levels were based on staining intensity and area of tumor cells.

2.4. Flow Cytometric Sorting. Cancer cells were isolated from the esophagus of tamoxifen-induced BmilCreER;Rosa ${ }^{t d T o-}$ mato mice through combining mechanical dissociation with enzymatic degradation by Tumor Dissociation kits (Miltenyi, 130-096-730). To be specific, 0.04-1 g tumor tissue was cut into small pieces of $2-4 \mathrm{~mm}^{3}$ and was dissociated in a volume of approximately $2.5 \mathrm{~mL}$ enzyme mix. Then, isolated cells were filtered through a $75 \mu \mathrm{m}$ diameter mesh and centrifuged at $300 \times \mathrm{g}$ for 7 minutes and supernatant was aspirated completely. Then, cells were washed with $1 \times$ PBS and resuspended with $90 \mu \mathrm{L} 1 \times$ PBS per $10^{7}$ total cells. Then, antiEpCAM (1:500; Abcam, ab221552) was added and reaction solution was incubated for 30 minutes on ice. Cells were washed and resuspended in $500 \mu \mathrm{L} 1 \times$ PBS and incubated with a secondary antibody ( $1: 1000$; Abcam, ab6717) for 25 minutes on ice. Then, cell sorting was performed on $\mathrm{BD}$ FACSAria II. EpCAM ${ }^{+}$Tomato $^{+}$cells were collected. All steps were performed under sterile conditions.

2.5. Tumorsphere Formation Assays. Cells were seeded in 6well ultralow attachment plates at 3,000 cells/mL in stem cell medium as previously described [18]. Cells were cultured in a humidified $5 \% \mathrm{CO}_{2}$ incubator at $37^{\circ} \mathrm{C}$ for 7-10 days, during 


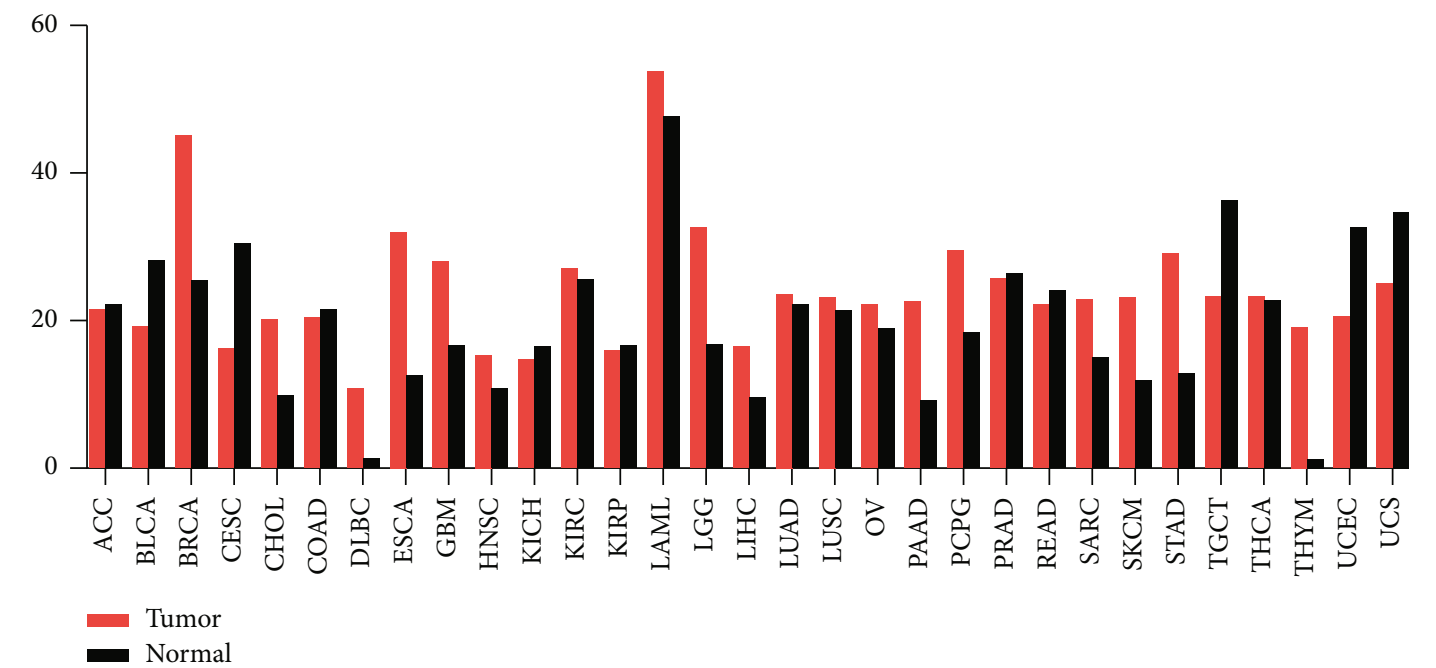

(a)

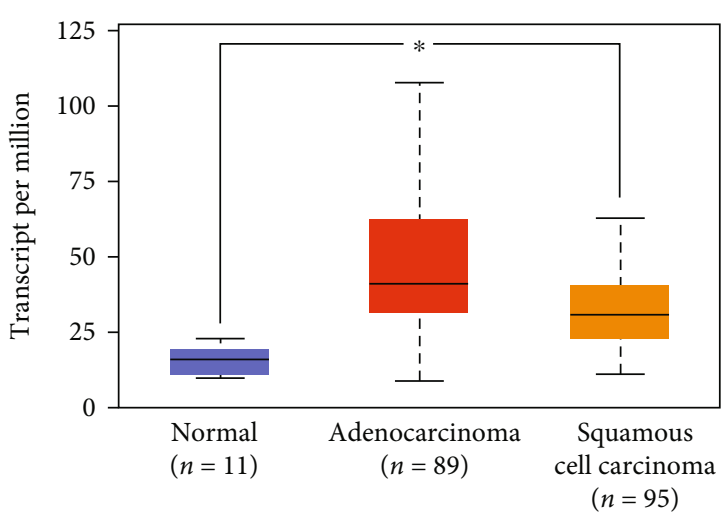

(b)

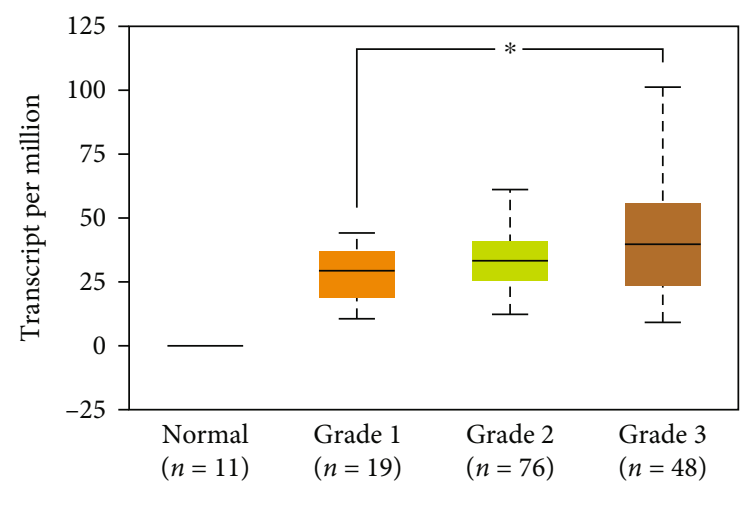

(c)

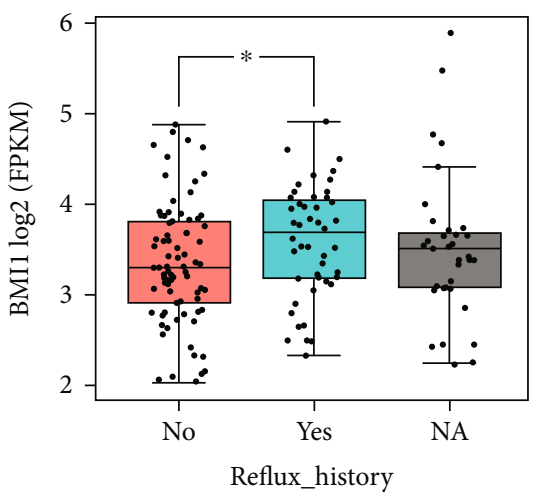

(d)

FIgURE 1: High expression of Bmil in esophageal carcinoma. (a) The TCGA database demonstrated that Bmil gene expression was significantly upregulated in esophageal carcinoma tissues. (b) Bmil expression levels in ESCA based on tumor histology were compared with normal tissues in the TCGA database $(n=195)$. (c) Bmil expression levels in different grades of esophageal carcinoma from TCGA database. (d) Gastroesophageal reflux is more likely to occur in patients with high expression of Bmil. No = patients without gastroesophageal reflux; Yes $=$ patients with gastroesophageal reflux; NA $=$ data missing. ${ }^{*} p<0.05$.

which serum-free media were changed every other day until the spheres formed. Then, tumorspheres were collected, washed with $1 \times$ PBS, and incubated with Trypsin-EDTA for two minutes at $37^{\circ} \mathrm{C}$. Then, the number of tumorspheres was counted. Three dishes were used for each group and all experiments were repeated three times.
2.6. TUNEL Assays. TUNEL assays are carried out using a commercial kit (KeyGEN, KGA703). According to the instructions provided by the manufacturer, freezing sections were fixed in $4 \%$ paraformaldehyde fix solution at room temperature $\left(15-25^{\circ} \mathrm{C}\right)$ for $20-30$ minutes and rinsed in $1 \times \mathrm{PBS}$ three times for 15 minutes. Then, sections were treated with 

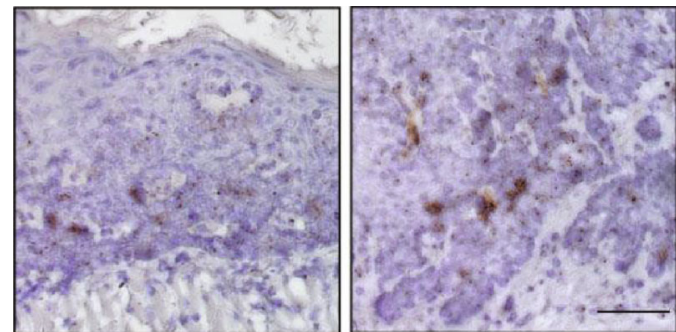

(a)

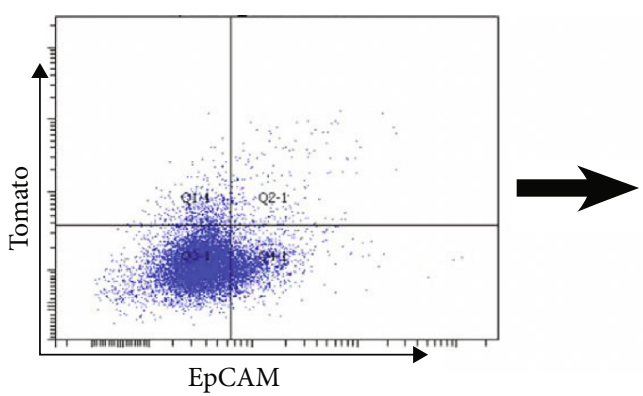

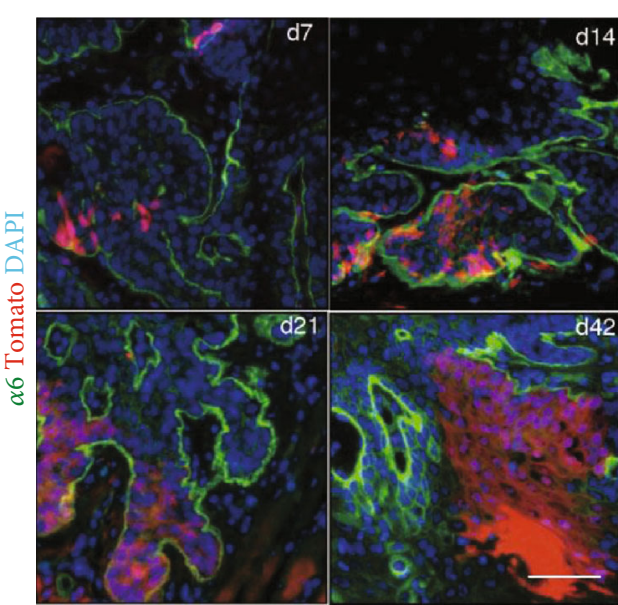

(b)

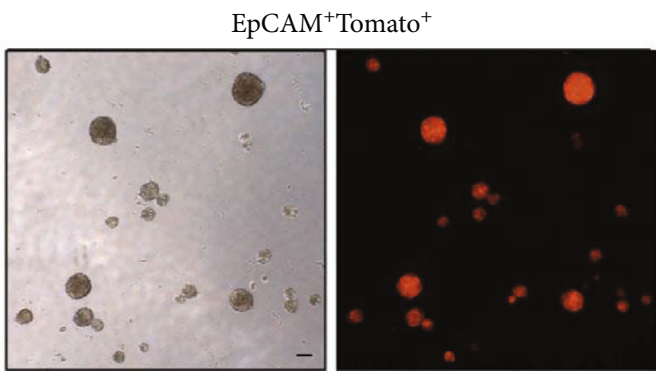

(c)

FIGURE 2: Bmi1 ${ }^{+}$cells and their progeny cells can self-renew in vivo and in vitro. (a) In situ hybridization of Bmil in ESCC samples induced by 4 NQO for 15 weeks (left) and 22 weeks (right). The brown area is the expression signal of the Bmil gene. (b) Bmil ${ }^{+}$cells are labeled in BmilCreER;Rosat ${ }^{\text {tdTomato }}$ mice following a single dose of tamoxifen and traced for 7, 14, 21, and 42 days. Red represents Tomato-positive Bmil-expressing cells and their differentiated progeny cells. Green labels integrin $\alpha 6$, a marker of the epithelial basement membrane. Nuclei are labeled in blue. (c) Three days after tamoxifen injection, EpCAM ${ }^{+}$Tomato $^{+}$double-positive cells sorted by flow cytometry were cultured into tumorspheres in vitro. Scale bars are $100 \mu \mathrm{m}$.

$100 \mu \mathrm{L}$ proteinase $\mathrm{K}$ for 30 minutes at $37^{\circ} \mathrm{C}$ and rinsed in 1 $\times$ PBS three times for 15 minutes again. After being immersed in $3 \% \mathrm{H}_{2} \mathrm{O}_{2}$ sealing liquid for 10 minutes and rinsed, the slides were added with $100 \mu \mathrm{L}$ DNase I reaction liquid containing 2000-3000 U, 40-60 $\mu \mathrm{L}$ DNase I (50 U/ $\mu \mathrm{L})$, and 60-40 $\mu \mathrm{L}$ DNase I buffer and rinsed. Later, TdT enzyme reaction solution, streptavidin-HRP working fluid, and DAB working fluid were added $50 \mu \mathrm{L}$ after each time the slides were rinsed in $1 \times$ PBS three times for 15 minutes and drained with blotting paper. Dyed in hematoxylin stain for 30 seconds to 5 minutes and washed with distilled water, the slides were put in methanol hydrochloride solution for differentiation for 5 seconds and washed with distilled water again, followed by $70 \%, 85 \%, 95 \%$, and absolute ethyl alcohol each for 5-minute rinse and xylene twice for ten minutes. After being dried, the samples were added with neutral balsam (Macklin, 822941-100 g), covered with glass slides, photographed, and observed under an optical microscope.

2.7. In Situ Hybridization. Dewaxed paraffin sections were obtained by the same method as $\mathrm{H} \& \mathrm{E}$. Incubation with $0.3 \% \mathrm{H}_{2} \mathrm{O}_{2}$ at room temperature for 30 minutes was done to remove endogenous peroxidase activity. After washing with $\mathrm{ddH}_{2} \mathrm{O}$, proteinase $\mathrm{K}$ was dripped onto paraffin sections and incubated at $37^{\circ} \mathrm{C}$ for 25 minutes. Then, the probe-free hybridization solution was added to pre-treat the slice (this step can be omitted). After washing with $\mathrm{ddH}_{2} \mathrm{O}$, the probe-containing hybridization solution was added and incubated at $46^{\circ} \mathrm{C}$ overnight. Sections were soaked three times with $46^{\circ} \mathrm{C} \mathrm{ddH_{2 }} \mathrm{O}$ for 15 minutes each. Peroxidaselabeled sheep anti-digoxin antibody was added and incubated at room temperature for 2 hours. Observation under an optical microscope after color development with DAB was done.

2.8. Statistical Analysis. The two-tailed unpaired Student $t$-test was used to measure statistical significance. Data were presented as mean $\pm \mathrm{SD}$. All the data statistical analysis is carried out through GraphPad Prism 8.0. For an alpha probability of 0.05 , the sample size needed to detect statistical significance difference is at least 6 animals in each group.

\section{Results}

3.1. Expression Levels and Clinical Value of Bmi1 in Esophageal Carcinoma. According to the data of The Cancer 


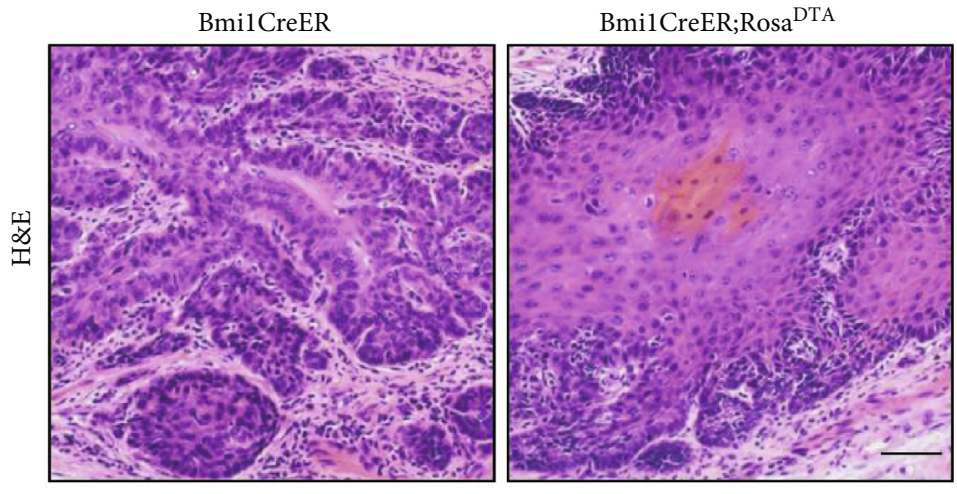

(a)
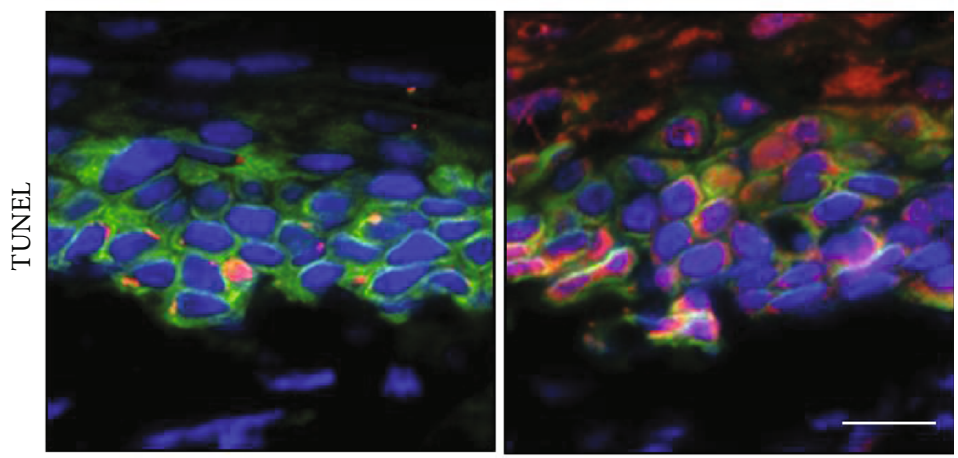

(b)

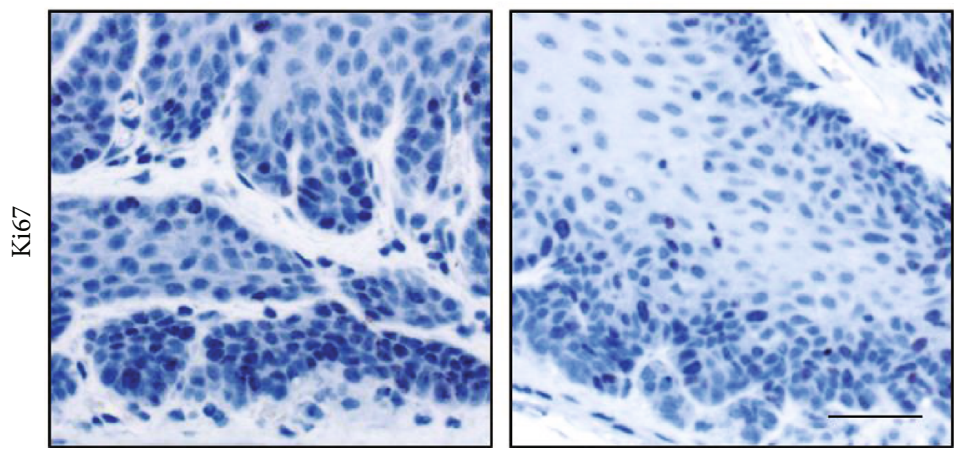

(c)
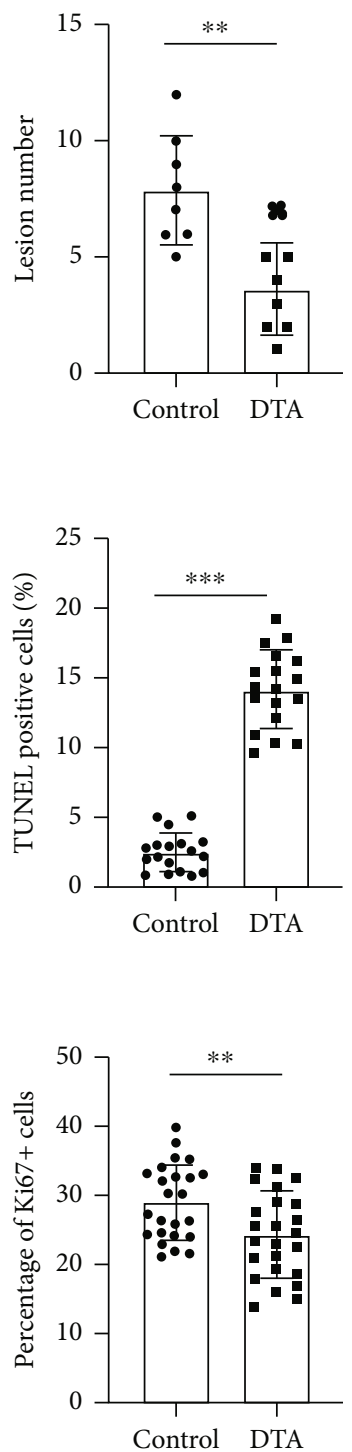

FIGURE 3: Depletion of $\mathrm{Bmil}^{+}$cells led to decreased proliferation and increased apoptotic phenotype in ESCC. (a) BmilCreER;Rosa ${ }^{\text {tdTomato }} ;$ Rosa $^{D T A}$ mice were compared with the control in the malignant degree of ESCC. (b) Apoptosis of the BmilCreER;Rosa ${ }^{\text {tdTomato }} ;$ Rosa $^{D T A}$ group was higher than that of the control group under the TUNEL test. Red indicates apoptotic cells. (c) Ki67 signal was reduced in ESCC without $\mathrm{Bmil}^{+}$cells, and Ki67 signal was represented by black. ${ }^{*} p<0.05,{ }^{* *} p<0.01$, and ${ }^{* * *} p<0.001$. Scale bars are $100 \mu \mathrm{m}$.

Genome Atlas (TCGA) database, the expression level of Bmil mRNA in esophageal carcinoma (ESCA) was upregulated in the esophageal carcinoma tissues compared with normal tissues (Figure 1(a)). And both ESCC and EAC tissues' Bmilexpression levels were significantly higher than normal tissues (Figure 1(b)). In addition, the expression of Bmil was correlated with the tumor grade of ESCA; although there were marginal differences between grades 1 and 2 and grades 2 and 3, there was still significant difference between grades 1 and 3 (Figure 1(c)). Furthermore, the clinical phenotype of Bmil expression pattern demonstrated that patients with gastroesophageal reflux tended to express higher Bmil leve1 (Figure 1(d)). And gastroesophageal reflux was considered to be one of the precancerous diseases of esophageal cancer [28]. Overall, these data indicated the potential clinical significance of Bmil in esophageal cancer patients.

3.2. $\mathrm{Bmil}^{+}$Cells Are the TICs in Mouse ESCC. To investigate whether Bmil is expressed in the mouse ESCC, we performed in situ hybridization assay. Our results indicate that Bmil is expressed in the subsets of ESCC cells in the areas near the basement membrane 15 weeks and 22 weeks after 4NQO treatment (Figure 2(a)), suggesting a possible role of Bmil-expressing cells. To verify whether Bmil-expressing 
cells have a role in ESCC, we bred Bmi1CreER;Rosa ${ }^{\text {tdTomato }}$ transgenic mice and carried out lineage tracing assay. Our data showed that a small number of Tomato ${ }^{+}$cells were presented near the basement membrane 7 days after tamoxifen injection. In the samples taken at 14 days, 21 days, and 42 days, the number of Tomato ${ }^{+}$cells increased with time and distributed in both the epithelial basal layer and the epithelial interstitial layer. Especially on the 42nd day, Tomato $^{+}$cells were widely distributed in the esophageal epithelium (Figure 2(b)). This shows that in ESCC, the offspring cells differentiated from $\mathrm{Bmil}^{+}$cells located in the basement membrane gradually develop into tumor parenchyma cells of ESCC over time. To further probe the stemness of $\mathrm{Bmil}^{+}$ cells, we harvested ESCC mice three days after injection of tamoxifen and isolated $\mathrm{EpCAM}^{+} \mathrm{Tomato}^{+}$double-positive cells for sphere formation assay. We found that ESCC $\mathrm{Bmil}^{+}$cells could form spheres in vitro (Figure 2(c)). Together, these data showed that the $\mathrm{Bmil}^{+}$cells served as TICs in mouse ESCC.

3.3. Bmil ${ }^{+}$Cells Are Critical for ESCC Progression. In order to explore the role of $\mathrm{Bmil}^{+}$cells in the progression of ESCC, we also treated BmilCreER;Rosa ${ }^{\text {tdTomato }}$; Rosa ${ }^{D T A}$ mice with $4 \mathrm{NQO}$. At 16 weeks of $4 \mathrm{NQO}$ treatment, $\mathrm{Bmil}^{+}$cells underwent apoptosis caused by diphtheria toxin after tamoxifen injection, resulting in almost no $\mathrm{Bmil}^{+}$cells in the tumor. After the removal of $\mathrm{Bmil}^{+}$cells, we can see that the lesion number was reduced (Figure 3(a)). Our results also showed that after the removal of $\mathrm{Bmil}^{+}$cells, the proportion of apoptosis of ESCC cells increased and the ability of proliferation was decreased (Figures 3(b) and 3(c)). These results indicate that $\mathrm{Bmil}^{+}$cells are important for the growth of ESCC.

3.4. The Expression of ESCC TIC Markers Was Inhibited after $\mathrm{Bmil}^{+}$Cell Ablation. Further, we examined the expression of known ESCC TIC markers, CD44, tp63, and KRT5, in ESCC tissue after $\mathrm{Bmil}^{+}$cell removal. Our data showed that Bmil ${ }^{+}$ cells in ESCC were almost eliminated after tamoxifen administration (Figure 4(a)). In the absence of Bmil ${ }^{+}$cells, we found that the expression of three tumor stem cell markers (CD44, Tp63, and Krt5) decreased in ESCC (Figures 4(b)-4(d)). It shows that after the scarcity of $\mathrm{Bmil}^{+}$cells, the stemness characteristics of ESCC are largely inhibited, suggesting that Bmil is important for the stemness of ESCC.

3.5. Cisplatin Combined with PTC-209 in the Treatment of ESCC. Our previous studies have proved that Bmil is an important tumor stem cell marker of ESCC, so the targeted therapy of $\mathrm{Bmil}^{+}$cells may provide a possibility for the clinical treatment of ESCC. Based on the above data, we carried out the animal drug experiment of cisplatin combined with PTC-209 (a small molecular inhibitor of Bmil). The experimental results show that although cisplatin and PTC-209 alone have a certain therapeutic effect, the combination of cisplatin and PTC-209 has the least lesion number and the best therapeutic effect (Figures 5(a) and $5(b))$.

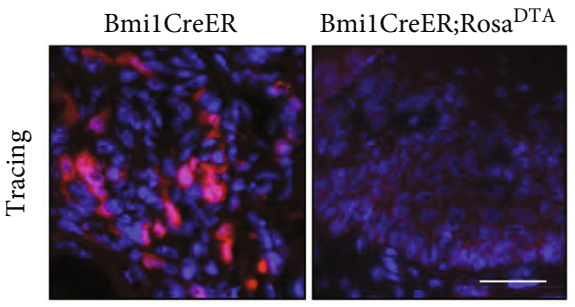

(a)

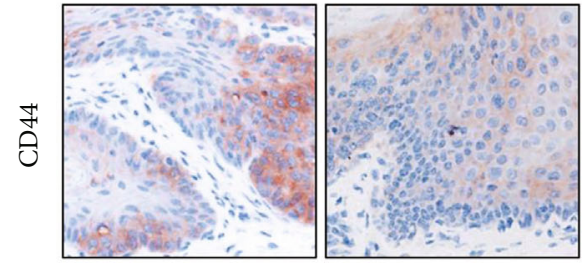

(b)

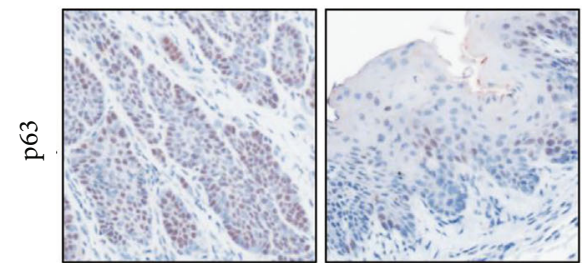

(c)

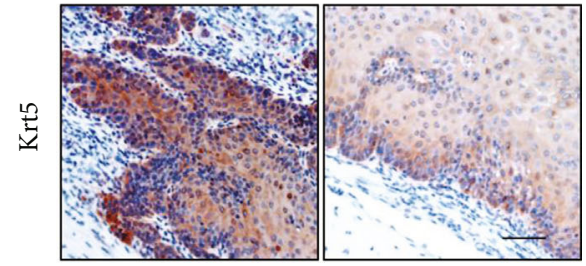

(d)

FIgUre 4: The stemness phenotype of ESCC without $\mathrm{Bmil}^{+}$cells was inhibited. (a) Samples of control and BmilCreER; Rosa ${ }^{\text {tdTomato }} ;$ Rosa ${ }^{D T A}$ mice were collected on the 21 st day after a single intraperitoneal injection of tamoxifen. Bmi1 ${ }^{+}$cells of BmilCreER;Rosa ${ }^{\text {tdTomato }} ;$ Rosa $^{D T A}$ mice were effectively removed. Red labels $\mathrm{Bmil}^{+}$cells and their progenies. (b-d) In ESCC samples, all three types of TIC markers, CD44 (b), tp63 (c), and $\mathrm{Krt5}$ (d), were reduced after $\mathrm{Bmil}^{+}$cells were removed. Brown represents the signals of interest. Scale bars are $100 \mu \mathrm{m}$.

\section{Discussion}

Bmil plays the important part in maintaining the dynamic balance of mitochondrial function and redox and can function like stem cells and progenitor cells to regulate cellular metabolism [29]. Notably, despite the key role of Bmil in the self-renewal of various somatic cancer stem cells that has been reported [30], there is no research focusing on Bmil in ESCC. In our study, we first confirmed the high expression of Bmil in esophageal carcinoma and patients with high expression prone to malignant transformation. After that, we generated BmilCreER;Rosa ${ }^{\text {tdTomato }}$ transgenic mice and explored the role of $\mathrm{Bmil}^{+}$cells as a source of carcinogenesis in ESCC. We utilized genetic lineage tracing analysis [31] and identified a subpopulation of $\mathrm{Bmil}^{+}$tumor cells that give rise to progressively growing epithelial clones. 

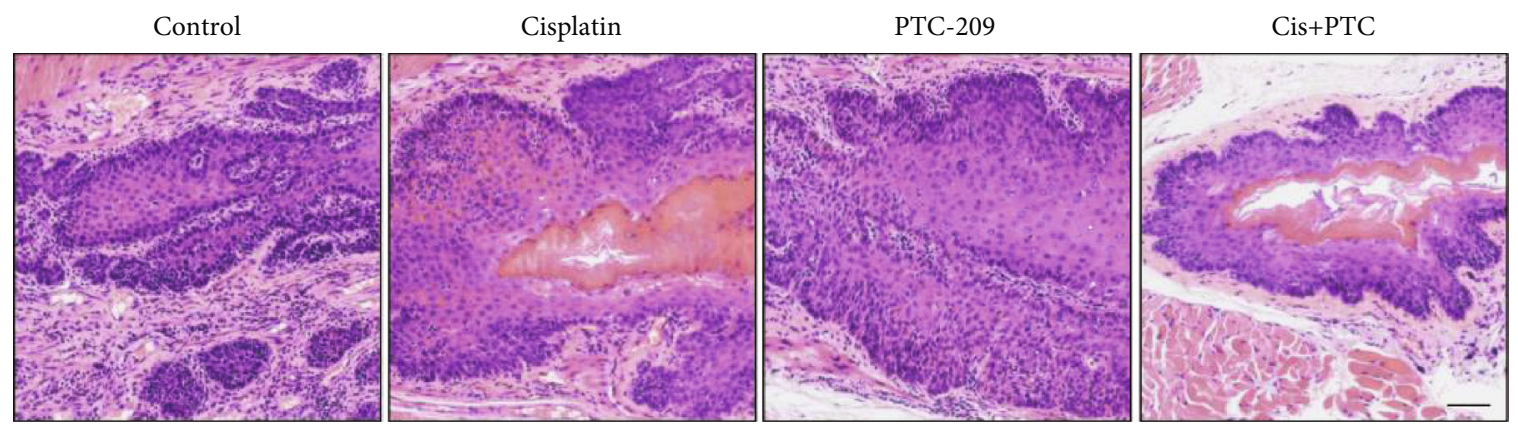

(a)

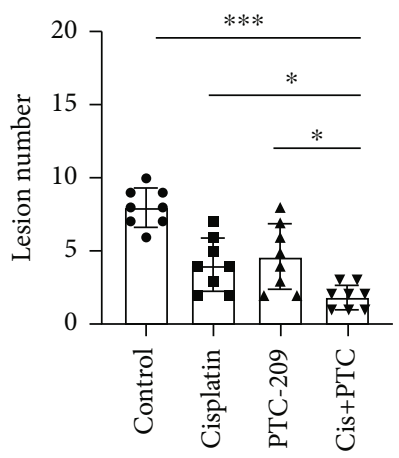

(b)

Figure 5: Treatment effect of cisplatin combined with PTC-209 on ESCC. (a, b) From left to right, the histopathology (a) and histogram (b) of ESCC in the control group, cisplatin-treated group, PTC-209-treated group, and the cisplatin ${ }^{+}$PTC-209-treated group. The processing time of the four groups was four weeks. Cisplatin combined with PTC-209 has the lowest malignancy. ${ }^{*} p<0.05,{ }^{* *} p<0.01$, and ${ }^{* * *} p<0.001$. Scale bars are $100 \mu \mathrm{m}$.

In addition, we demonstrated that genetic ablation of Bmil resulted in increased apoptosis and decreased proliferation. We also compared different treatment strategies of ESCC, and we found that the therapeutic effect of targeting both the tumor bulk and TICs was better than monotherapy [32-34].

The esophagus has a keratinized squamous epithelium consisting of four to five cell layers with rapid turnover characteristics, which is maintained by proliferative basal cells. These basal cells can renew, differentiate, and migrate to the lumen, producing the upper basal layer of terminally differentiated cells. In previous studies, clonal analysis using CreER transgenic mice suggests that Sox2- and K15-labeled progenitor and/or stem cell populations have a higher potential for self-renewal than that of committed progenitors [35, 36]. In the present study, Bmil actively expressed in the ESCC and Bmil ${ }^{+}$cells were enriched in ESCC with stem cell properties. Importantly, $\mathrm{Bmil}^{+}$cells differentiated into a large chunk of the tumor of the BmilCreER;Rosa ${ }^{\text {tdTomato }}$ mouse over time, consistent with previous studies [37, 38]. Moreover, $\mathrm{Bmil}^{+}$cell depletion by using BmilCreER;Rosa ${ }^{\text {td }}$ ${ }^{\text {Tomato }}$;Rosa ${ }^{\text {DTA }}$ mouse further proves that $\mathrm{Bmil}^{+}$cells function as TICs in ESCC.

In terms of the treatment of esophageal cancer, the effect of monotherapy is not all satisfactory. Cisplatin is capable of killing proliferative cells [39] but has limited effect on relatively resting cells such as TICs, resulting in poor efficacy. Our study showed that $\mathrm{Bmil}^{+}$cells are endowed with malignant phenotype. We treated mouse with ESCC using mono- therapy or combined therapy and analyzed the treated tumors. We found that targeting both the tumor bulk and $\mathrm{Bmil}^{+}$cells achieved the most efficacious tumor control. Consistently, a recent study on glioblastomas shows that combined Bmil targeting and another molecular targeting drug proved more effective than either agent alone both in culture and in vivo [32]. In addition, Bmil inhibitor PTC209 has been proved to inhibit tumor growth by targeting CSC self-renewal in head and neck squamous cell carcinoma [18]. Our results demonstrate the significant efficacy of combined inhibition of Bmil and cisplatin, but we did not detect cures: the efficacy against each subtype was preferential, but not absolute. Future studies will determine how to achieve the desired results.

\section{Conclusions}

To sum up, our data provide specific experimental evidence that $\mathrm{Bmil}^{+}$cells are the cell origin of ESCC. Monotherapy alone against ESCC tumor growth near the limits of detection and combinational therapy of Bmil inhibitor and cisplatin were the most effective in reducing tumor burdens. Although the molecular basis of Bmil-derived carcinogenesis and the clinical significance of Bmil-derived ESCC have yet to be further studied, exploring the carcinogenic mechanism of multiple malignant tumors from the perspective of cell origin will provide us with new and promising therapeutic strategies. 


\section{Data Availability}

The data used to support the findings of this study are available from the corresponding author upon request.

\section{Conflicts of Interest}

The authors indicate no potential conflicts.

\section{Authors' Contributions}

Xiaochen Wang and Kang Li contributed equally to this work.

\section{Acknowledgments}

This paper was supported by grants from the Natural Science Foundation of Guangdong Province (no. 2018A030313610) and the National Natural Science Foundation of China (no. 81872409).

\section{References}

[1] F. Bray, J. Ferlay, I. Soerjomataram, R. L. Siegel, L. A. Torre, and A. Jemal, "Global cancer statistics 2018: GLOBOCAN estimates of incidence and mortality worldwide for 36 cancers in 185 countries," CA: a Cancer Journal for Clinicians, vol. 68, no. 6, pp. 394-424, 2018.

[2] E. C. Smyth, J. Lagergren, R. C. Fitzgerald et al., "Oesophageal cancer," Nature Reviews Disease Primers, vol. 3, no. 1, p. 17048 , 2017.

[3] R. L. Siegel, K. D. Miller, and A. Jemal, "Cancer statistics, 2018," CA: a Cancer Journal for Clinicians, vol. 68, no. 1, pp. 7-30, 2018.

[4] J. Chen, Y. Li, T. S. Yu et al., "A restricted cell population propagates glioblastoma growth after chemotherapy," Nature, vol. 488, no. 7412, pp. 522-526, 2012.

[5] M. Najafi, K. Mortezaee, and R. Ahadi, "Cancer stem cell (a)symmetry \& plasticity: tumorigenesis and therapy relevance," Life Sciences, vol. 231, p. 116520, 2019.

[6] E. Batlle and H. Clevers, "Cancer stem cells revisited," Nature Medicine, vol. 23, no. 10, pp. 1124-1134, 2017.

[7] T. Lapidot, C. Sirard, J. Vormoor et al., "A cell initiating human acute myeloid leukaemia after transplantation into SCID mice," Nature, vol. 367, no. 6464, pp. 645-648, 1994.

[8] S. K. Singh, C. Hawkins, I. D. Clarke et al., "Identification of human brain tumour initiating cells," Nature, vol. 432, no. 7015, pp. 396-401, 2004.

[9] A. T. Collins, P. A. Berry, C. Hyde, M. J. Stower, and N. J. Maitland, "Prospective identification of tumorigenic prostate cancer stem cells," Cancer Research, vol. 65, no. 23, pp. 10946-10951, 2005.

[10] S. Zhang, C. Balch, M. W. Chan et al., "Identification and characterization of ovarian cancer-initiating cells from primary human tumors," Cancer Research, vol. 68, no. 11, pp. 43114320, 2008.

[11] J. S. Zhao, W. J. Li, D. Ge et al., "Tumor initiating cells in esophageal squamous cell carcinomas express high levels of CD44," PLoS One, vol. 6, no. 6, article e21419, 2011.

[12] T. N. Almanaa, M. E. Geusz, and R. J. Jamasbi, "A new method for identifying stem-like cells in esophageal cancer cell lines," Journal of Cancer, vol. 4, no. 7, pp. 536-548, 2013.
[13] K. H. Tang, Y. D. Dai, M. Tong et al., “A CD90(+) tumorinitiating cell population with an aggressive signature and metastatic capacity in esophageal cancer," Cancer Research, vol. 73, no. 7, pp. 2322-2332, 2013.

[14] Q. Liu, X. Cui, X. Yu et al., "Cripto-1 acts as a functional marker of cancer stem-like cells and predicts prognosis of the patients in esophageal squamous cell carcinoma," Molecular Cancer, vol. 16, no. 1, p. 81, 2017.

[15] X. Y. Ming, L. Fu, L. Y. Zhang et al., “_Integrin $\alpha 7_{-}$is a functional cancer stem cell surface marker in oesophageal squamous cell carcinoma," Nature Communications, vol. 7, no. 1, article 13568, 2016.

[16] B.-B. S. Zhou, H. Zhang, M. Damelin, K. G. Geles, J. C. Grindley, and P. B. Dirks, "Tumour-initiating cells: challenges and opportunities for anticancer drug discovery," Nature Reviews. Drug Discovery, vol. 8, no. 10, pp. 806-823, 2009.

[17] N. Goto, A. Fukuda, Y. Yamaga et al., "Lineage tracing and targeting of IL17RB(+) tuft cell-like human colorectal cancer stem cells," Proceedings of the National Academy of Sciences of the United States of America, vol. 116, no. 26, pp. 1299613005, 2019.

[18] D. Chen, M. Wu, Y. Li et al., "Targeting BMI1 ${ }^{+}$Cancer Stem Cells Overcomes Chemoresistance and Inhibits Metastases in Squamous Cell Carcinoma," Cell Stem Cell, vol. 20, no. 5, pp. 621-634.e6, 2017.

[19] Y. Miao, H. Yang, J. Levorse et al., "Adaptive immune resistance emerges from tumor-initiating stem cells," Cell, vol. 177, no. 5, pp. 1172-1186.e14, 2019.

[20] B. Storti, S. Civita, P. Faraci et al., "Fluorescence imaging of biochemical relationship between ubiquitinated histone $2 \mathrm{~A}$ and Polycomb complex protein BMI1," Biophysical Chemistry, vol. 253, article 106225, 2019.

[21] B. Biehs, J. K. H. Hu, N. B. Strauli et al., "BMI1 represses _Ink4a/Arf_and_Hox_ genes to regulate stem cells in the rodent incisor," Nature Cell Biology, vol. 15, no. 7, pp. 846852, 2013.

[22] R. Ferretti, A. Bhutkar, M. C. McNamara, and J. A. Lees, "BMI1 induces an invasive signature in melanoma that promotes metastasis and chemoresistance," Genes \& Development, vol. 30, no. 1, pp. 18-33, 2016.

[23] H. Ren, P. du, Z. Ge et al., "TWIST1 and BMI1 in cancer metastasis and chemoresistance," Journal of Cancer, vol. 7, no. 9, pp. 1074-1080, 2016.

[24] J. Liu, K. Liu, X. Jiang et al., "Clinicopathological significance of Bmi-1 overexpression in esophageal cancer: a meta-analysis," Biomarkers in Medicine, vol. 12, no. 1, pp. 71-81, 2018.

[25] Y. W. Xu, Y. H. Peng, B. Chen et al., “Autoantibodies as potential biomarkers for the early detection of esophageal squamous cell carcinoma," The American Journal of Gastroenterology, vol. 109, no. 1, pp. 36-45, 2014.

[26] H. Kojima, T. Okumura, T. Yamaguchi, T. Miwa, Y. Shimada, and T. Nagata, "Enhanced cancer stem cell properties of a mitotically quiescent subpopulation of p75NTR-positive cells in esophageal squamous cell carcinoma," International Journal of Oncology, vol. 51, no. 1, pp. 49-62, 2017.

[27] S. Saboor-Maleki, F. B. Rassouli, M. M. Matin, and M. Iranshahi, "Auraptene attenuates malignant properties of esophageal stem-like cancer cells," Technology in Cancer Research \& Treatment, vol. 16, no. 4, pp. 519-527, 2016.

[28] D. Arcidiacono, A. Dedja, C. Giacometti et al., "Hyperinsulinemia promotes esophageal cancer development in a surgically- 
induced duodeno-esophageal reflux murine model," International Journal of Molecular Sciences, vol. 19, no. 4, p. 1198, 2018.

[29] J. Liu, L. Cao, J. Chen et al., "Bmil regulates mitochondrial function and the DNA damage response pathway," Nature, vol. 459, no. 7245, pp. 387-392, 2009.

[30] L. L. Smith, J. Yeung, B. B. Zeisig et al., "Functional crosstalk between Bmil and MLL/Hoxa9 axis in establishment of normal hematopoietic and leukemic stem cells," Cell Stem Cell, vol. 8, no. 6, pp. 649-662, 2011.

[31] S. S. Wu, J. H. Lee, and B. K. Koo, "Lineage tracing: computational reconstruction goes beyond the limit of imaging," Molecules and Cells, vol. 42, no. 2, pp. 104-112, 2019.

[32] X. Jin, L. J. Y. Kim, Q. Wu et al., "Targeting glioma stem cells through combined BMI1 and EZH2 inhibition," Nature Medicine, vol. 23, no. 11, pp. 1352-1361, 2017.

[33] E. V. Schmidt, "Developing combination strategies using PD-1 checkpoint inhibitors to treat cancer," Seminars in Immunopathology, vol. 41, no. 1, pp. 21-30, 2019.

[34] A. K. Nooka and S. Lonial, "Novel combination treatments in multiple myeloma," Oncology, vol. 30, no. 5, pp. 451-465, 2016.

[35] K. Arnold, A. Sarkar, M. A. Yram et al., "Sox $2^{+}$Adult Stem and Progenitor Cells Are Important for Tissue Regeneration and Survival of Mice," Cell Stem Cell, vol. 9, no. 4, pp. 317-329, 2011.

[36] V. Giroux, A. A. Lento, M. Islam et al., "Long-lived keratin 15+ esophageal progenitor cells contribute to homeostasis and regeneration," The Journal of Clinical Investigation, vol. 127, no. 6, pp. 2378-2391, 2017.

[37] K. Liu, M. Jiang, Y. Lu et al., "Sox2 cooperates with inflammation-mediated Stat3 activation in the malignant transformation of foregut basal progenitor cells," Cell Stem Cell, vol. 12, no. 3, pp. 304-315, 2013.

[38] J. Frede, P. Greulich, T. Nagy, B. D. Simons, and P. H. Jones, "A single dividing cell population with imbalanced fate drives oesophageal tumour growth," Nature Cell Biology, vol. 18, no. 9, pp. 967-978, 2016.

[39] S. Dasari and P. B. Tchounwou, "Cisplatin in cancer therapy: molecular mechanisms of action," European Journal of Pharmacology, vol. 740, pp. 364-378, 2014. 